\title{
Advances in Research on Biomedical Nanomaterials in Vietnam
}

\author{
Bich Ha Nguyen* \\ Institute of Materials Science, Vietnam Academy of Science and Technology, Vietnam
}

Received: 觜 May 01, 2018; Published: 阱 May 14, 2018

*Corresponding author: Bich Ha Nguyen, Institute of Materials Science, Vietnam Academy of Science and Technology, 18 Hoang Quoc Viet, Hanoi, Vietnam; Email: bichha@iop.vast.ac.vn

\begin{abstract}
The present article is a concise review on the application - oriented basic research on nanomedicine in Vietnam during the 5-year period 2012-2016. The content of review includes following topics: biomedical utilization of targeting folate-decorated paclitaxel-loaded PLA-TPGS nanomaterial, dendrimer-based anticancer drug, special drug delivery nanosystems, various utilization of nanocurcumin in nanomedicine, biosensor and biosensing methods, and antibacterial activity of several types of nanoparticles.
\end{abstract}

\section{Introduction}

Application-oriented basic research on biomedical nanomaterials in Vietnam started 6 years ago, in the year 2012. During 5-year period 2012-2016 Vietnamese scientists achieved promising research results in following topics: paclitaxel-loaded PLA-TPGS nanomaterial, doxorubicin- loaded PLA-TPGS nanomaterial, curcumin-loaded PLA-TPGS nanomaterial, dendrimer-based anticancer drug, special drug delivery systems, biosensors and biosensing methods, toxicity and antibacterial activity of several types of metallic nanoparticles. The present work is a review of the articles of Vietnamese scientists on biomedical nanomaterials published in the journal Advances in Natural Sciences: Nanoscience and Nanotechnology during five years 2012-2016.

\section{Scientific Achievements}

Only one year 2012 eleven articles [1-11] have been published. In reference Ha P T et al. [1] presented the results of their research on paclitaxel-loaded poly(lactide)-d- $\alpha$-tocopheryl polyethylene glycol 1000 succinate (PLA-TPGS) nanoparticles prepared by a modified solvent extraction/evaporation technique. The authors experimentally demonstrated the ability of paclitaxel-loaded PLATPGS nanoparticles to induce apoptosis in human hepatocellular carcinoma cell lines (Hep-G2), indication the potential application of these nanoparticles as a cancer chemotherapeutic agent. In reference Dang M C et al. [2] reported on the fabrication of a nanoparticle formulation of ketoprofen (Keto)-encapsulated cucurbit[6] (CB[6]) uril nanoparticles, the evaluation of its in vitro dissolution and the investigation of its in vivo pharmaceutical property. The CB [6]-Keto nanoparticles were prepared by emulsion solvent evaporation method. Morphology and size of the successfully prepared nanoparticles were then confirmed using a transmission electron microscope and dynamic light scattering. It was shown that they are spherical with hydrodynamic diameter of 200-300 $\mathrm{nm}$. The in vitro dissolution studies of $\mathrm{CB}[6]-$ Keto nanoparticles were conducted at $\mathrm{pH} 1.2$ and 7.4. The results indicated that there is a significant increase in Keto concentration at $\mathrm{pH} 7.4$ compared to $\mathrm{pH}$ 1.2. For the in vivo assessment $\mathrm{CB}[6]$-Keto nanoparticles and referential profenid were administered by oral gavages to rabbits. The results implied that $\mathrm{CB}[6]-$ Keto nanoparticles remarkably increased the area under the curve compared to profenid.

A curcumin-loaded polylactic acid nanofiber constructed by electrospinning was investigated by Nguyen X P et al. [3]. Curcumin (Cur) extracted from the Curcuma longa L. plant is well-known for its anti-tumor, anti-oxidant, anti-flammatory and anti-bacterial properties. Nanofiber mats of polylactic acid (PLA) loading Cur (5 wt\%) were fabricated by electrospinning (e-spinning). The diameter of the obtained fibers varied from 200 to $300 \mathrm{~nm}$. The release capacity of curcumin from curcuminloaded PLA fibers was investigated in phosphate buffered saline (PBS) containing ethanol. After $24 \mathrm{~h}, 50 \%$ of the curcumin was released from curcumin-loaded PLA fibers. The results concerning electrospun fibers exhibit the potential for biomedical application. Polymer-encapsulated curcumin nanoparticles were prepared and investigated by Nguyen X P et al. [4]. It is well-known that curcumin (Cur) is a yellow compound isolated from rhizome of 
the herb curcumin longa. Curcumin processes anti-oxidant, antiflammatory, anti-carcinogenic and anti-microbial properties. It also suppresses proliferation of many tumor cells. However, the clinical application of curcumin in cancer treatment is considerably limited due to its serious poor delivery characteristics. In order to increase the hydrophilicity and drug delivery capability the authors encapsulated curcumin into copolymer PLA-TPGS, 1,3- $\beta$-glucan (Glu), 0-carboxymethyl chitosan (OCMCS) and folate-conjunged OCMCS (OCMCS-Fol).

These polymer-encapsulated curcumin nanoparticles (Cur-PLATPGS, Cur-Glu, Cur-OCMCS and Cur-OCMCS-Fol) were characterized by infrared, fluorescence, photoluminescence spectroscopy, field emission scanning electron microscopy, and found to be spherical particles with an average size 50-100 nm. They were much more soluble in water than not only free curcumin but also other biodegradable polymer-encapsulated curcumin nanoparticles. The anti-tumor promoting assay was carried out, showing the positive effects of Cur-Glu and Cur-PLA-TPGS on tumor promotion of Hep-G2 cell line in vitro. Confocal microscopy revealed that the nano-sized curcumin encapsulated by OCMCS and OCMCS-Fol significantly enhanced the cellular uptake (cancer cell HT29 and HeLa).

Biosensor for cholesterol detection using interdigitated electrodes based on polyaniline-carbon nanotube film was fabricated by Tran DL et al. [5]. In this work polyaniline-carboxylic multiwalled carbon nanotube composite film (PANi-MWCNT) has been polymerized on the surface of interdigitated platinum electrode (fabricated by MEMS technology) which was compatibly connected to Autolab interface via universal serial bus. An amperometric biosensor based on covalent immobilization of cholesterol oxidase (ChOx) on PANi-MWCNT film with potassium ferricyanide (FeCN) as the redox mediator was developed. The mediator helps to shuttle the electrons between the immobilized ChOx and the PANi-MWCNT electrode, therefore operating at a low potential $-0.3 \mathrm{~V}$ compared to the saturated calomel electrode. This potential precludes the interfering compounds from oxidation. The bio-electrode exhibits good linearity from 0.02 to $1.2 \mathrm{mM}$ cholesterol concentration with a correlation coefficient of 0.9985 .

Electrochemical immunosensors based on different serum anti-body immobilization methods for detecting Japanese encephalitis virut (JEV) was developed by Tran Q H et al. [6]. Human serum containing anti-JEV was immobilized onto the surface of silanized interdigitated electrodes by four methods: direct adsorption (APTES-serum), covalent binding with a cross linker of glutaraldehyde (APTES-GA-serum), covalent binding with a cross linker of glutaraldehyde combined with anti-human IgG (APTES-GA-anti-HIgG-serum) and covalent binding with a cross linker of glutaraldehyde combined with a bioaffinity of protein A (APTES-GA-PrA-serum). Atomic force microscopy was used to verify surface characteristics of the interdigitated electrodes before and after treatment with serum antibodies. The output signal of the immunosensors was measured with the change of conductivity resulting from the specific binding of JEV antigens and serum antibodies immobilized on the electrodes with the help of horseradish peroxidax (HRP)-labeled secondary anti-body against JEV. In reference Le QM et al. [7] presented the results of the preparation of the luminescent lanthanide nanophosphore (LLN) and bioconjugates linked with LLN as well as their application as label tool for recognizing virus in the processing line of vaccine industrial production. Several LLNs with the nanostructure forms of particles or rods/wires with europium (III) and terbium (III) ions in lattices of vanadate, phosphate and metal organic complex were prepared to develop novel fluorescent conjugates able to be applied as labels in fluorescence immunoassay analysis of virus/ vaccine.

Graphene patterned polyaniline-based biosensor for glucose detection was fabricated by Phan N M et al. [8]. In this work a glucose electrochemical biosensor was layer-by-layer fabricated from graphene and polyaniline films. Graphene sheets $\left(0.5^{*} 0.5\right.$ $\mathrm{cm}^{2}$ ) with the thickness of $5 \mathrm{~nm}$ (15 layers) were synthesized by thermal chemical vapor deposition under ambient pressure on cooper tapes. Then they were transferred into integrated $\mathrm{Fe}_{3} \mathrm{O}_{4}$ doped polyaniline (PANi) based microelectrodes. The properties of the nanocomposite films were characterized by scanning electron microscopy (SEM), Raman spectroscopy, atomic force microscopy (AFM) and electrochemical methods such as square wave voltammetry (SWV) and chronoamperometry. The prepared graphene patterned sensor (denoted as Graphene $/ \mathrm{Fe}_{3} \mathrm{O}_{4} / \mathrm{PANi}$ / GOx) shows much improved glucose sensitivity (as high as $47 \mu \mathrm{A}$ $\mathrm{M}^{-1} \mathrm{~cm}^{-2}$ ) compared to sensors without graphene (10-30 $\mu \mathrm{A} \mathrm{mM}^{-1}$ $\left.\mathrm{cm}^{-2}\right)$.

Dye-doped water soluble silica-based nanoparticles to lebel bacteria E. coli 0157:H7 were investigated by Tran H N etal. [9]. In this work organically modified silicate (ORMOSIL) nanoparticles (NPs) doped with rhodamine $6 \mathrm{G}$ and rhodamine $\mathrm{B}$ dyes were synthesized by Stöber method from methytrietho-xylane $\mathrm{CH}_{3} \mathrm{Si}\left(\mathrm{OCH}_{3}\right)_{3}$ precursor (MTEOS). The NPs are surface functionalized by cationic amino groups. The optical characterization of dye-doped ORMOSIL NPs was studied in comparison with that of free dye in solution. The synthesized NPs were used for labeling bacteria E. coli 0157:H7. The number of bacteria has been counted using the fluorescence spectra and microscope images. The results show the ability of NPs to work as biomarkers.

Capping and in vivo toxicity studies of gold nanoparticles were performed by Tran $\mathrm{H} \mathrm{N}$ et al. [10]. In this work water-dispersed colloidal gold nanoparticles (AuNPs) with high concentration were synthesized from metal precursor $\mathrm{HAuCl}_{4}$. The bovine serium albumin (BSA) and heterobiofunctionalized thiol polyethylene glycol acid (HS-PEG-COOH) were used as biofunctionalized layers for the synthesized AuNPs. The BSA and HS-PEG-COOH bound to the AuNPs were characterized qualitatively and quantitatively by transmission electron microscope (TEM) and UV-Vis spectrophotometer. The fabricated BSA and HS-PEG-COOH- caped AuNPs were introduced in mouse to study its toxicity and its availability in the liver. 
In reference Vu DL et al. [11] have demonstrated the powerful disinfectant ability of colloidal silver nanoparticles (AgNPs) for the prevention of gastrointestinal bacterial infections. AgNPs colloid was synthesized by a UV-enhanced chemical precipitation. Two gastrointestinal bacterial strains of Escherichia coli (ATCC 43888-0157:k-:H7) and Vibrio cholera (01) were used to verify the antibacterial activity of the as-prepared AgNPs colloid by means of surface disinfection assay in agar plates and turbidity assay in liquid media. Transmission electron microscopy was also employed to analyze the ultrastructural changes.

The demonstration of a high efficiency for loading and releasing dendrimer-based anticancer drugs against cancer cell in vitro and in vivo was performed by Nguyen T P et al. [12]. In this work pegylated polyamidoamine (PAMAM) dendrimer at generation 3.0 (G 3.0) and carboxylated PAMAM dendrimer G 2.5 were prepared for loading anticancer drugs. For loading cisplatin, carboxylated dendrimer could carry 26.64 wt $\%$ of ciplatin. The nanocomplexes have size ranging from 10 to $30 \mathrm{~nm}$ in diameter. The drug nanocarrier showed activity against NCI-H 460 lung cancer cell line with IC50 of $23.11 \pm 2.08 \mu \mathrm{g} \mathrm{ml}^{-1}$. Pegylated PAMAM dendrimer (G 3.0) were synthesized below $40 \mathrm{~nm}$ in diameter for carrying 5 -fluorouracil (5-FU). For 5-FU encapsulation, pegylated dendrimer showed a high drug-loading efficiency of the drug and a slow release profile of 5-FU. The drug nanocarrier system exhibited an antiproliferative activity against MCF-7 cells with a $\mathrm{IC}_{50}$ of $9.92 \pm 0.19 \mu \mathrm{g} \mathrm{ml}^{-1}$. In vivo tumor xemograft study showed that the 5-FU encapsulated pegylation of dendrimer exhibited a significant decrement in volume of tumor which was generated by MCF-7 cancer cells.

Curcumin-loaded pluronic F127/chitosan nanoparticles for cancer therapy were prepared by Le Q $\mathrm{H}$ et al. [13]. In this work curcumin-loaded nanoparticles have been prepared by an ionic gelation method using chitosan (CS) and pluronic F127 (PF) as carriers to deliver curcumin to the target cancer cells. Prepared nanoparticles were characterized using zetasizer, fluorescence microscopy, SEM and TEM. The results showed that the encapsulation efficiency of curcumin was approximately $50 \%$. The average size of curcumin-loaded PF/CS nanoparticles was $150.9 \mathrm{~nm}$, while the zeta potential was $5.09 \mathrm{mV}$. Cellular uptake of curcumin-loaded nanoparticles into HEK293 cells was confirmed by fluorescence microscopy.

The fabrication of layer-by-layer biosensor using graphene films and the application for cholesterol determination were performed by Tran D L et al. [14]. The graphene films were synthesized by thermal chemical vapor deposition method. Methane gas $\left(\mathrm{CH}_{4}\right)$ and cooper tape were used as carbon source and catalyst in the graphene growth process, respectively. The intergrated array was fabricated by using micro-electro-mechanical system (MEMS) technology in which $\mathrm{Fe}_{3} \mathrm{O}_{4}$-doped polyaniline (PANi) film was electropolymerized on $\mathrm{Pt} / \mathrm{Gr}$ electrodes. The properties of the $\mathrm{Pt} / \mathrm{Gr} / \mathrm{PANi} / \mathrm{Fe}_{3} \mathrm{O}_{4}$ films were investigated by field emission scanning electron microscopy (FESEM), Raman spectroscopy and electrochemical technique. Cholesterol oxidase (ChOx) has been immobilized onto the working electrode with glutaraldehyde agent. The cholesterol electrochemical biosensor show high sensitivity $\left(74 \mu \mathrm{A} \mathrm{mM}{ }^{-1} \mathrm{~cm}^{\text {- }}\right.$ $\left.{ }^{2}\right)$ and fast response time ( $<5 \mathrm{sec}$ ). A linear calibration plot was obtained in the wide cholesterol concentration range from 2 to 20 $\mathrm{mM}$ and correlation coefficient square $\left(\mathrm{R}^{2}\right)$ of 0.9986 .

Electrosynthesis of polyaniline-multiwalled carbon nanotube nanocomposite film for glucose biosensing was performed by Nguyen $\mathrm{T}$ D et al. [15]. In this work polyaniline-multiwalled carbon nanotube (PANi-MWCNT) nanocomposites were electropolymerized in the presence of sodium dodecyl sulfate (SDS) onto interdigitated platinum film plannar microelectrodes $(\mathrm{IP} \mu \mathrm{E})$. The MWCNTs were first dispersed in SDS solution then mixed with aniline and $\mathrm{H}_{2} \mathrm{SO}_{4}$. This mixture was used to electrosynthesize PANi-MWCNT films with potentiostatic method at $\mathrm{E}=$ $+0.9 \mathrm{~V}$ (versus SCE). The PANi-MWCNT films were characterized by cyclic voltammetry (CV) and SEM. The results show that the PANi-MWCNT films have a high electroactivity and a porous and branched structure that can increase the specific surface area for biosensing application. The PANi-MWCNT films were applied for covalent immobilization of glucose oxidase (GOx) via glutaraldehyde agent. The GOx/PANi-MWCNT/ID $\mu \mathrm{E}$ was studied using cyclic voltammetric and chronoamperometric techniques. The effect of several interferences, such as ascorbic acid (AA), uric acid (UA) and acetaminophen (AAP) on the glucose sensing at +0.6 $\mathrm{V}$ (versus SCE) is not significant. The time required to reach $95 \%$ of the maximum steady-state current was less than $5 \mathrm{sec}$.

In reference Tran N Q et al. [16] introduced tetronic grafted chitosan containing tyramide moieties which have been utilized for in situ enzyme-mediated hydrogel preparation. The hydrogel can be used to load nanoparticles of biphastic calcium phosphate (BCP), mixture of hydroxyapatite (Hap) and tricalcium photsphate (TCP), forming injectable biocomposites. The grafted copolymers were well-characterized by proton nuclear magnetic resonance (1H-NMR). BCP nanoparticles were prepared by precipitation method under ultrasonic irradiation then characterized by using XRD and SEM. The suspension of the copolymer and BCP nanoparticles rapidly formed hydrogel biocomposite with a few seconds of the presence of horseradish peroxidase (HRP) and $\mathrm{H}_{2} \mathrm{O}$. In vitro study using mesenchyman stem cells showed that the composites were biocompatible and cells were well-attached on the surface.

In reference Ngo V K T et al. [17] demonstrated a quartz crystal microbalance (QCM) as biosensor for detecting E. coli 0157:H7. The E. coli 0157:H7 antibodies were immobilized on a self-assembly monolayer (SAM) modified $5 \mathrm{MHz}$ AT-cut quartz crystal resonator. The SAMs were activated with 16-mercaptopropanoic acid, in the presence of 1-ethyl-3-(3-dimethylaminopropyl)carbodiimide (EDC) and N-hydroxysuccinimide (NHS). The results of changing frequency due to the adsorption of E. coli 0157:H7 was measured by the QCM biosensor system designed and fabricated by ICDREC- 
VNUHCM. This system gave good results in the range of $10^{3}-10^{7} \mathrm{CFU}$ $\mathrm{ml}^{-1}$ E. coli 0157:H7. The time of bacteria E. coli 0157:H7 detection in the sample was about 50 minutes. Besides, QCM biosensor from SAM method was comparable to protein A method-based piezoelectric immunosensor in terms of the amount of immobilized antibodies and detection sensitivity.

In reference Ha P $\mathrm{T}$ et al. [18] studied enhanced cellular uptake and cytotoxicity of folate-decorated doxorubicin (DOX)loaded PLA-TPGS nanoparticles. DOX is one of the most effective anticancer drugs for treating many types of cancer. However, the clinical application of DOX was hindered because of serious side-effects resulting from the unselective delivery to cancer cell including congestive heart failure, chronic cardiomyopathy and drug resistance. Recently it has been demonstrated that loading anticancer drugs onto drug delivery nanosystems help to maximize therapeutic efficiency via passive and active targeting mechanisms. In this work the authors prepared folate-decorated DOX-loaded PLA-TPGS nanoparticles with the aim of improving the potential as well as reducing the side-effects of DOX. Anticancer activity of the nanoparticles was evaluated through cytotoxicity and cellular uptake assays on HeLa and HT29 cancer cell lines. Their material characteristics were investigated by field emission scanning electron microscopy (FESEM), dynamic light scattering (DLS) and Fourrier transform infrared spectroscopy (FTIR). The results showed that prepared drug delivery system had size around 100 $\mathrm{nm}$ and exhibited higher cytotoxicity and cellular uptake on both HeLa and HT29 cells.

As a new copolymer material for oral delivery of insulin Dang M C et al. [19] used poly(ethylene glycol)-grafted chitosan. The authors proposed a new scheme of grafting poly(ethylene glycol) onto chitosan (CS). First, methoxy poly(ethylene glycol) amine (mPEGa MW 2000) were grafted onto CS through multiple steps to synthesize the grafting copolymer PEG-g-CS. After each synthesis step CS and its derivatives were characterized by FTIR and ${ }^{1} \mathrm{H}-\mathrm{NMR}$. Then insulin-loaded PEG-g-CS nanoparticles were prepared by cross-liking of CS with sodium tripolyphosphate (TPP). Same insulin-loaded nanoparticles using unmodified CS were also prepared in order to compare with the modified one. Results showed better protecting capacity of the synthesized copolymer over original CS. CS nanoparticles (19 nm of size) were gel like and highly sensible to temperature as well as acidic environment while PEG-g-CS nanoparticles ( $200 \mathrm{~nm}$ of size) were rigit and more thermo and $\mathrm{pH}$ stable.

A significant progress in the research on silica-based optical nanoparticles for biomedical applications was achieved by Tran $\mathrm{H}$ $\mathrm{N}$ et al. [20]. This article is a review of their research results. Gold, dye-doped silica-based and core-shell multifunctional multilayer $\left(\mathrm{SiO}_{2} / \mathrm{Au}, \quad \mathrm{Fe}_{3} \mathrm{O}_{4} / \mathrm{SiO}_{2}, \quad \mathrm{Fe}_{3} \mathrm{O}_{4} / \mathrm{SiO}_{2} / \mathrm{Au}\right)$ water monodispersed nanoparticles were synthesized by chemical route and surface modified with protein and biocompatible chemical agents. The particles were conjungated with antibody or aptamer for specific detecting and imaging bacteria and cancer cells. The photothermal effects of gold nanoparticles $\left(\mathrm{SiO}_{2} / \mathrm{Au}\right.$ and $\left.\mathrm{Fe}_{3} \mathrm{O}_{4} / \mathrm{SiO}_{2} / \mathrm{Au}\right)$ on cells and tissues were investigated. Moreover, the nano silver substrates were also developed for surface enhanced Raman scattering (SERS) spectroscopy to detect melamine.

The preparation of gold nanoparticles by microwave heating and the study of their conjugates with E. coli 0157:H7 antibodies were demonstrated by Ngo V K T et al. [21]. In this article the authors described a method for the low coast synthesis of gold nanoparticles using sodium citrate $(\mathrm{Na} 3 \mathrm{Ct})$ reduction in chloroauric acid (HAuCl4.3H2O) by microwave heating. Gold nanoparticles were functionalized with surface activation by 3-mercaptopropionic acid for attaching antibodies. These nanoparticles were then reacted with E. coli 0157:H7 antibodies using N-hydroxysuccinimide and carbodiimide hydrochloride coupling chemistry. The product was characterized by UV-Vis spectroscopy, FTIR, Zeta potential measurement and TEM. The diameters of nanoparticles have the values in the range 13-15 $\mathrm{nm}$. Moreover, the TEM images demonstrated the binding of gold nanoparticles with the antibodies.

Silicon nanowire sensor for detecting alpha-fetoprotein biomarker of live cancer was fabricated by Dang MC et al. [22]. In this work the authors applied a facile method using only conventional microtechniques and two size-reduction steps to fabricate wafer - scale silicon nanowire (SiNW) with the width of $200 \mathrm{~nm}$. Initially conventional lithography was used to pattern SiNW with $2 \mu \mathrm{m}$ width. Then the nanowire width was decreased to $200 \mathrm{~nm}$ by two size-reduction steps with isotropic etching. The electrical characteristics of fabricated SiNW were investigated. Then a simple and effective surface modification process was performed for subsequent binding to desired receptor, and the complete SiNW-based biosensor was fabricated for using to detect alpha-ketoprotein (AFP), one of the medically approved biomarkers for liver cancer diagnosis. Electrical measurements showed that the fabricated SiNW-based biosensor could detect AFP with concentration of about $100 \mathrm{ng} \mathrm{ml-1}$, which is appropriate for liver cancer diagnosis.

Silver chloride nanoparticles as an antibacterial agent were investigated by Nguyen T H et al. [23]. In this work silver chloride nanoparticles were prepared by the precipitation reation between silver nitrate and sodium chloride in an aqueous solution containing poly(vinyl alcohol) as a stabilizing agent. Different characteristics of the nanoparticles in suspension and in lyophilized powder such as size, morphology, chemical nature, interaction with stabilizing agent and photostability were investigated. Biological tests showed that the obtained silver chloride nanoparticles displayed antibacterial activities against Escherichia coli and Staphylococcus aureus. Targeted drug delivery nanosystems based on TPGS for cancer treatment were investigated by Ha P T et al. [24]. Along with the development of nanotechnology, drug delivery nanosystems (DDNSs) have attracted a great deal of concern among scientists over the world, especially in cancer treatment. DDNSs not only 
improve water solubility of anticancer drugs but also increase therapeutic efficacy and minimize the side effects of treatment methods through targeting mechanisms including passive and active targeting. Passive targeting is based on the nanosize of drug delivery nanosystems while active targeting is based on the specific bindings between targeting ligands attached on the drug delivery nanosystems and the unique receptors on the cancer cell surface. In this article the authors present some results in the synthesis and testing of DDNSs prepared from copolymer poly(lactide)tocopheryl polyethylene glycol succinate (PLA-TPGS), which carry anticancer drugs including curcumin, paclitaxel and doxorubicin. In order to increase the targeting effect to cancer cells, active targeting ligand folate was attached to the DDNSs. The results showed copolymer PLA-TPGS to be an excellent carrier for loading hydrophobic drugs (curcumin and paclitaxel). The fabricated DDNSs had a very small size $(50-100 \mathrm{~nm})$ and emhanced the cellular uptake and cytotoxicity of drugs. Most notably, folate-decorated paclitaxel-loaded copolymer PLA-TPGS nanoparticles (Fol/PTX/ PLA-TPGS NPs) were tested on tumor-bearing nude mice. During the treatment time, Fol/PTX/PLA-TPGS NPs always exhibited the best tumor growth inhibition compared to free paclitaxel.

Curcumin as fluorescence probe for directly monitoring in vitro uptake of curcumin-combined paclitaxel-loaded PLA-TPGS was studied by Hoang T M N et al. [25]. It was well-known that theranostics, which is the combination of both therapeutic and diagnostic capacities in one dose, is a promising tool for both clinical application and research. Although there are many chromophores available for optical imaging, their application are limited due to the photobleaching property or intrinsic toxicity. Curcumin, a natural compound extracted from the rhizome of curcuma longa, is well-known thanks to its bio-pharmaceutical activities and strong fluorescence as biocompatible for bio-imaging.

In this work the authors prepared composite nanoparticles with dual functions: dianogstic and therapeutic, based on poly(lactide)-tocopheryl polyethylene glycol succinate (PLA-TPGS) micelles co-loaded by curcumin (Cur) and paclitaxel (PTX). These composite nanoparticles were characterized by FESEM and DLS methods. Their cellular uptake and fluorescent activities were also tested by bioassay studies. Obtained results showed that they have promising potential for application in cancer theranostics. In vitro evaluation of Aurona kinase inhibitor VX 680 in formulation of PLATPGS nanoparticles was performed by Hoang T M N et al. [26]. In this work polymeric nanoparticles prepared from PLA-TPGS were used as potential drug carriers with many advantages to overcome the disadvantages of insoluble anticancer drugs and enhance blood circulation time. VX680 is an Aurona kinase inhibitor. The purpose of this work is to investigate whether VX680-loaded PLATPGS nanoparticles are able to effectively increase the toxicity of chemotherapy. Accordingly, the authors first synthesized VX680loaded nanoparticles and performed the characterizations of morphology, mean size, Zeta potential and encapsulation efficiency.
Then they investigated the effects on HeLa cells. The cell cytotoxicity was evaluated by the xCELLigence real-time cell analyser allowing measurement of changes in electrical impedence on the surface of the E-plate. Analysis of nuclear morphology and level of histone H3 phosphorylation was observed by confocal fluorescence scanning microscopy. Cell cycle distribution and apoptosis were analyzed by flow cytometry. The results showed that VX680-loaded PLATPGS nanoparticles reduced cell viability with IC50 value lower 3.4 times compared to free VX680. Thus VX680-loaded PLA-TPGS nanoparticles may be considered as promising drug delivery system for cancer treatment.

\section{Conclusion}

Application-oriented basic research on nanomedicine began in Vietnam about six years ago with the first publication in 2012. Since that time it rapidly developed and obtained promising results. Some of them were applied to the industrial production of several food supplements such as CURMAGOLD, CURMIN Nano 22, HEPOSAL B, FGC and KUMAR KUL.

Further development of above presented research works could be directed towards two directions:

a) Enrichment of the scientific contents of the basic research works.

b) Implementation of the research results both to the diagnosis as well as to the therapy of the diseases.

\section{References}

1. Hoai Nam Nguyen, Hong Ha Tran Thi, Duong Le Quang, Toan Nguyen Thi, Nhu Hang Tran Thi, et al. (2012) Apoptosis induced by paclitaxel-loaded copolymer PLA-TPGS in Hep-G2 cells. Adv. Nat Sci Nanosci Nanotechnol 3045005 .

2. Nguyen To Hoai, Phuong Tuyen Thi Dao, Quoc Nam Phu, Duy Dam Le, et al. (2012) Ketoprofen encapsulated cucurbit [6] uril nanoparticles: a new exploration of macrocycles for drug delivery. Adv Nat Sci Nanosci Nanotechnol 3045004.

3. Thi Thu Trang Mai, Thi Thu Thuy Nguyen, Quang Duong Le, Thi Ngoan Nguyen, Thi Cham Ba, et al. (2012) A novel nanofiber Cur-loaded polylactic acid constructed by electrospinning. Adv Nat Sci Nanosci. Nanotechnol 3025014.

4. Phuong Thu Ha, Mai Huong Le, Thi My Nhung Hoang, Thi Thu Huong Le, Tuan Quang Duong, et al. (2012) Preparation and anti-cancer activity of polymer-encapsulated curcumin nanoparticles. Adv Nat Sci Nanosci Nanotechnol 3035002.

5. Le Huy Nguyen, Hai Binh Nguyen, Ngoc Thinh Nguyen, Tuan Dung Nguyen, Dai Lam Tran (2012) Portable cholesterol detection with polyaniline-carbon nanotube film based interdigitated electrodes. Adv Nat Sci Nanosci Nanotechnol 3015004.

6. Quang Huy Tran, Anh Tuan Mai, Thanh Thuy Nguyen, Van Chung Pham, Thi Hong Hanh Nguyen (2012) Towards the use of protein A-tagged gold nanoparticles for signal amplification of electrochemical immunosensors in virus detection. Adv Nat Sci Nanosci Nanotechnol 3025013.

7. Quoc Minh Le, Thu Huong Tran, Thanh Huong Nguyen, Thi Khuyen Hoang, Thanh Binh Nguyen, et al. (2012) Development of a fluorescent label tool based on lanthanide nanophosphors for viral biomedical application. Adv Nat Sci Nanosci Nanotechnol 3035003. 
8. Hai Binh Nguyen, Van Chuc Nguyen, Van Tu Nguyen, Thi Thanh Tam Ngo, Ngoc Thinh Nguyen, et al. (2012) Graphene patterned polyanilinebased biosensor for glucose detection. Adv Nat Sci Nanosci. Nanotechnol 3025011.

9. Minh Tan Pham, Thi Van Nguyen, Thuy Duong Vu Thi, Ha Lien Nghiem Thi, Kim Thuan Tong, et al. (2012) Synthesis, photophysical properties and application of dye doped water soluble silica-based nanoparticles to label bacteria E. coli 0157:H7. Adv Nat Sci Nanosci Nanotechnol 3 045013.

10. Thi Ha Lien Nghiem, Thi Tuyen Nguyen, Emmanuel Fort, Thanh Phuong Nguyen, Thi My Nhung Hoang, et al. (2012) Capping and in vivo toxicity studies of gold nanoparticles. Adv Nat Sci Nanosci Nanotechnol 3 015002 .

11. Anh-Tuan Le, Thi Tam Le, Van Quy Nguyen, Huy Hoang Tran, Duc Anh Dang, Quang Huy Tran and Dinh Lam Vu (2012) Powerful colloidal silver nanoparticles for the prevention of gastrointestinal bacterial infections. Adv Nat Sci Nanosci Nanotechnol 3045007.

12. Ngoc Quyen Tran, Cuu Khoa Nguyen, Thi Phuong Nguyen (2013) Dendrimer-based nanocarriers demonstrating a high efficiency for loading and releasing anticancer drugs against cancer cells in vitro and in vivo. Adv Nat Sci Nanosci Nanotechnol 4045013.

13. Thi Minh Phuc Le, Van Phuc Pham, Thi Minh Lua Dang, Thi Huyen La, Thi Hanh Le, et al. (2013) Preparation of curcumin-loaded pluronic F127/chitosan nanoparticles for cancer therapy. Adv Nat Sci Nanosci Nanotechnol 4025001.

14. Hai Binh Nguyen, Van Chuc Nguyen, Van Tu Nguyen, Huu Doan Le, Van Quynh Nguyen, et al. (2013) Development of the layer-by-layer biosensor using graphene films: application for cholesterol determination. Adv Nat Sci Nanosci Nanotechnol 4015013.

15. Trong Huyen Le, Ngoc Thang Trinh, Le Huy Nguyen, Hai Binh Nguyen, Van Anh Nguyen, et al. (2013) Electrosynthesis of polyanilinemutilwalled carbon nanotube nanocomposite films in the presence of sodium dodecyl sulfate for glucose biosensing. Adv Nat Sci Nanosci Nanotechnol 4025014.

16. Thi Phuong Nguyen, Bach Hai Phuong Doan, Dinh Vu Dang, Cuu Khoa Nguyen and Ngoc Quyen Tran (2014) Enzyme-mediated in situ preparation of biocompatible hydrogel composites from chitosan derivative and biphasic calcium phosphate nanoparticles for bone regeneration. Adv Nat Sci Nanosci Nanotechnol 5015012.

17. Vo Ke Thanh Ngo, Dang Giang Nguyen, Hoang Phuong Uyen Nguyen, Van Man Tran, Thi Khoa My Nguyen, et al. (2014) Quartz crystal microbalance
(QCM) as biosensor for the detecting of Escherichia coli 0157:H7. Adv Nat Sci Nanosci Nanotechnol 5045004

18. Hoai Nam Nguyen, Thi My Nhung Hoang, Thi Thu Trang Mai, Thi Quynh Trang Nguyen, Hai Doan Do, et al. (2015) Enhanced cellular uptake and cytotoxicity of folate decorated doxorubicin loaded PLA-TPGS nanoparticles. Adv Nat Sci Nanosci Nanotechnol 6025005.

19. Thanh Ha Ho, Thi Nu Thanh Le, Tuan Anh Nguyen and Mau Chien Dang (2015) Poly(ethylene glycol) grafted chitosan as new copolymer material for oral delivery of insulin. Adv Nat Sci Nanosci Nanotechnol 6035004 .

20. Hong Nhung Tran, Thi Ha Lien Nghiem, Thi Thuy Duong Vu, Viet Ha Chu, Quang Huan Le, et al. (2015) Optical nanoparticles: synthesis and biomedical application. Adv Nat Sci Nanosci Nanotechnol 6023002.

21. Vo Ke Thanh Ngo, Hoang Phuong Uyen Nguyen, Trong Phat Huynh, Nguyen Nguyen Pham Tran, Quang Vinh Lam, et al. (2015) Preparation of gold nanoparticles by microwave heating and application of spectroscopy to study conjugate of gold nanoparticles with antibody $E$. coli 0157:H7. Adv Nat Sci Nanosci Nanotechnol 6035015.

22. Van Binh Pham, Xuan ThanhTung Pham, Thanh Nhat Khoa Phan, Thi Thanh Tuyen Le, Mau Chien Dang (2015) Facile fabrication of a silicon nanowire sensor by two size reduction steps for detection of alphafetoprotein biomarker of liver cancer. Adv Nat Sci Nanosci Nanotechnol 6045001.

23. Ngoc Duong Trinh, Thi Thanh Binh Nguyen and Thanh Hai Nguyen (2015) Preparation and characterization of silver chloride nanoparticles as an antibacterial agent. Adv Nat Sci Nanosci Nanotechnol 6045011.

24. Phuong Thu Ha, Hoai Nam Nguyen, Hai Doan Do, Quoc Thong Phan, Minh Nguyet Tran Thi, et al. (2016) Targeted drug delivery nanosystems based on copolymer poly(lactide)-tocopheryl polyethylene glycol succinate for cancer treatment. Adv Nat Sci Nanosci Nanotechnol 7015001.

25. Hoai Nam Nguyen, Phuong Thu Ha, Anh Sao Nguyen, Dac Tu Nguyen, Hai Doan Do, et al. (2016) Curcumin as fluorescent probe for directly monitoring in vitrouptake of curcumin combined paclitaxel loaded PLATPGS nanoparticles. Adv Nat Sci Nanosci Nanotechnol 7025001.

26. Thi Thuy Duong Le, Phuong Thu Ha, Thi Hai Yen Tran, Dac Tu Nguyen, Hoai Nam Nguyen, Van Khanh Bui and My Nhung Hoang (2016) In vitro evaluation of Aurora kinase inhibitor-VX680-in formulation of PLA-TPGS nanoparticles. Adv Nat Sci Nanosci Nanotechnol 7025010.

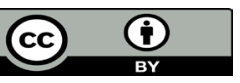

This work is licensed under Creative Commons Attribution 4.0 License

To Submit Your Article Click Here: Submit Article
DOI: 10.32474/OAJBEB.2018.02.000139

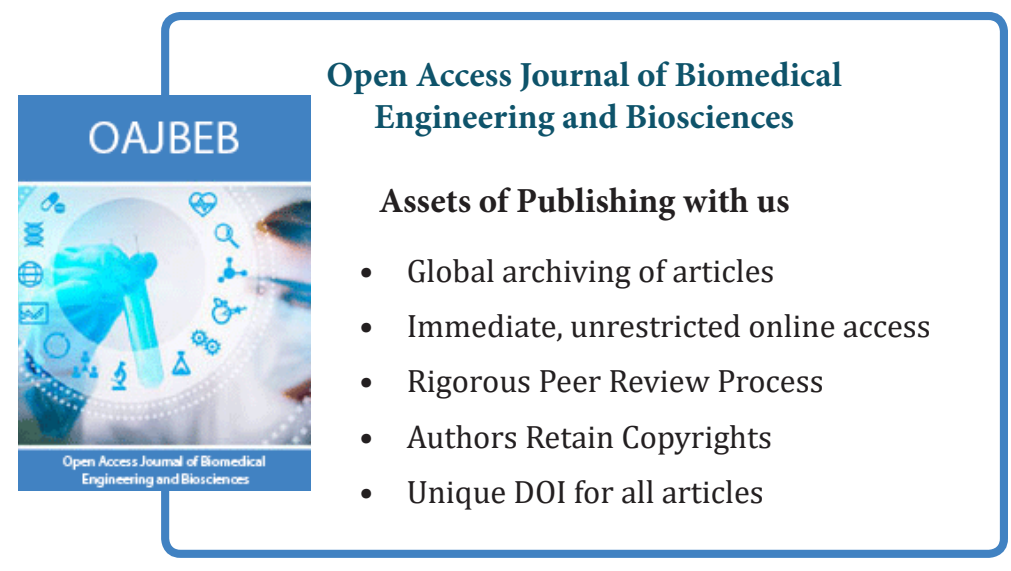

